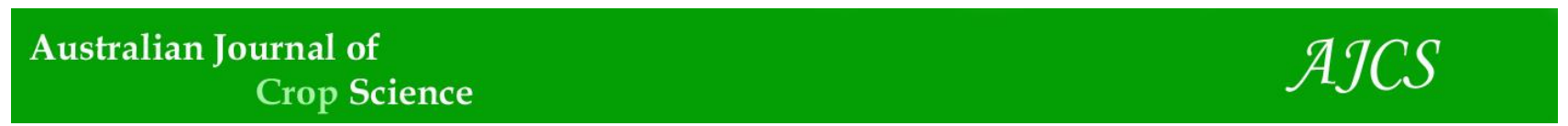

AJCS 14(07):1055-1063 (2020)

ISSN:1835-2707

doi: 10.21475/ajcs.20.14.07.p1924

\title{
Participatory variety selection of common bean (Phaseolus vulgaris L.) genotypes in the major bean producing areas of Ethiopia
}

\author{
Shiferaw G. Tigist1 ${ }^{1,2}$, Rob Melis ${ }^{2}$, Julia Sibiya ${ }^{2}$, Beyene Amelework ${ }^{3 *}$, Gemechu Keneni $^{4}$ \\ ${ }^{1}$ Ethiopian Institute of Agricultural Research, Melkassa Agricultural Research Centre, P.O. Box 430, Adama, Ethiopia. \\ ${ }^{2}$ African Center for Crop Improvement, University of KwaZulu-Natal, Private Bag X01, Scottsville 3209, \\ Pietermaritzburg, South Africa. \\ ${ }^{3}$ Agricultural Research Council, Vegetable and Ornamental Plant, Private Bag X293, Pretoria, 0001, South Africa. \\ ${ }^{4}$ Ethiopian Institute of Agricultural Research, Holeta Agricultural Research Centre, P.O.Box 2003, Addis Ababa, \\ Ethiopia.
}

\section{*Corresponding author: assefaa@arc.agric.za}

\section{Abstract}

This study was conducted to identify suitable parental genotypes for breeding for resistance to bruchid and to identify the farmers' preferred traits in common bean. For this study, 144 diverse common bean genotypes were planted in an alpha lattice design, with 3 replications at 3 locations. Participatory variety selection was done using 20 farmers at each location. Farmers identified a number of criteria for selecting suitable varieties. Yield and yield-related traits were ranked as the most important selection criteria by all farmers at all locations. However, women ranked culinary traits as the top criteria, while men were more interested in marketable traits. From the 144 genotypes, farmers selected the 10 best genotypes in all the locations. The majority of the genotypes selected at Melkassa and Alemetena were small white-seeded released varieties and breeding lines. However, all the genotypes selected at Arsi Negele were predominantly landraces of small red-seeded beans. Traits such as earliness and resistance to storage insect were important in Melkassa and Alme tena but received less attention in Arsi Negele. The integration of the farmers' selection preferences with the breeders' criteria can improve the efficiency of plant breeding by developing crop varieties that better fit the specific needs of the farmers.

Keywords: Phaseolus vulgaris, farmers' selection criteria, participatory variety selection.

Abbreviations: PVS_Participatory Variety Selection, CIAT_Center for Tropical Agriculture, m.a.s.I_meter above sea level, DAP_ Diammonium phosphate, RAZ_ Resistant to Zabrotes, MAZ_ Marker Assisted Zabrotes.

\section{Introduction}

The common bean (Phaseolus vulgaris L.) is the most important food legume in Ethiopia. The crop is cultivated in several agro-ecological zones and farming systems and mainly grown by small-scale farmers for household consumption, marketing and soil fertility improvement purposes (Asfaw et al., 2009, Asfaw et al., 2012; CSA, 2015). Ethiopian farmers have a higher preference to grow common beans, compared to other legumes, because they mature early, which helps them to obtain a cash income to buy food and other household needs. It also serves as an emergency crop in times of crop failure (Legesse et al., 2006). The common bean was introduced to Ethiopia in the sixteenth century and farmers have been able to adapt, develop and maintain a large genetic diversity to suit their needs. A range of bean types are grown in the country, but small white and red beans are the most common and preferred types. The small white beans are mainly grown in the Oromiya (in the Central Rift Valley) and Amhara regions, for the export market. Ethiopia exports white beans to the canning industry in Europe (Ferris and Kaganzi, 2008). The small red beans, on the other hand, are grown mainly in the southern parts of the country and they are used for local and regional markets and for household consumption (Ferris and Kaganzi, 2008; Rubyogo et al., 2011; CSA, 2015). Recently, due to the rising demand in the international and domestic market, the common bean is being grown in almost all parts of the country, with varying intensity (Katungi et al., 2009; CSA, 2015). Common bean production in the Central Rift Valley (Oromiya region) comprises about $50 \%$ of the total bean production of the country. Ninety-five percent of common bean-growing farmers produce the small white beans (Legesse et al., 2006; CSA, 2015). In Ethiopia, the National Common Bean Research Program plays an important role in meeting the increasing demand for the crop by releasing improved common bean varieties. Starting in the 1970s, the National Bean Program has developed and released more than 55 common bean varieties. Even though strong efforts have been made to disseminate these varieties, using different extension channels, the adoption rate has been slow, mainly due to the inaccessibility of improved seed (Pan Africa Bean Research Alliance, 2005; Dawit and Spielman, 2010; Buruchara et al., 2011). Over the past fifteen years, the national bean research program, in collaboration with the International Center for Tropical 
Agriculture (CIAT) has been working on the decentralization of the seed systems. Consequently, a dramatic increase in the area of production and productivity of the common bean has been observed in the country. Between 2004 and 2012, the area for common bean production significantly increased from 181,600 to 330,000 ha and the total production tripled to 387,000 tons per year. The average yield also increased from 0.62 to $1.50 \mathrm{t} / \mathrm{ha}$ (CIAT, 2013). Although considerable efforts have been made to improve the productivity of the crop in the country, there is still a huge gap between the potential and actual yield (Rubyogo et al., 2011; CIAT, 2013). Among the 55 improved varieties, only $18 \%$ were disseminated and adopted (Ferris and Kaganzi, 2008). The main reason for the poor adoption rate and low impact of the improved varieties are mainly due to the technological, socio-economical or agro-ecological constraints. Moreover, less coordinated efforts of the research and extension activities and poor integration of the farmers-consumerstraders value chain have by and large affected both the process and the outcome. To improve technology generation, dissemination and adoption, and to benefit from the available improved technologies, the different stakeholders (researchers, extension officers, farmers, consumers and traders) have to be part of the breeding process right from its inception. This can be done through participatory plant breeding in the identification of priority traits, on-farm demonstrations, popularization and reevaluation of the technologies (Ceccarelli et al., 2000; Ceccarelli and Grando, 2007). Participatory variety selection (PVS) is a powerful tool that involves farmers and other stakeholders to help orient breeding programs and to improve variety adoption (Sperling et al., 2001). It also assists plant breeders to develop technologies that fit into a specific production niche and the farmers' needs (Ceccarelli et al., 2000). The conventional plant breeding scheme uses a narrow range of selection criteria that addresses issues related to yield, uniformity and stability. Traditional farmers, however, employ more diverse and complex selection criteria, revolving around stable crop performance over seasons and they grow a range of genotypes that meet their needs in very complex and heterogeneous environments (Sperling and Loevinsohn, 1996; Ceccarelli and Grando, 2007). The farmers' preferences, as well as the socioeconomic aspects, are often ignored by the conventional breeding programs. Farmer participation in setting breeding goals and varietal evaluation will remain critical for enhancing adoption and genetic diversity. PVS can speed up the selection and fast-track the dissemination processes. In addition, it will eliminate a number of unacceptable varieties and save money and time (Mekbib, 1997; Assefa et al., 2006). The participatory evaluation of diverse common bean genotypes and the selection of parental material will be of paramount importance in designing possible improvement strategies, based on the farmers' priorities. Therefore, this study was carried out to evaluate diverse common bean genotypes, to identify suitable parental genotypes useful for breeding for bruchid resistance and to identify the farmers' selection criteria for choosing varieties.

\section{Results}

\section{Farmers' preference in variety selection}

Farmers attending the participatory variety selection had different selection criteria for each gender group and location. In the focus group discussion, both men and women farmers were able to list 14 selection criteria. There were some traits listed by men and not by women. The ranking of the criteria was different for men and women, as well as for the different locations (Table 1). However, some similarities in the selection criteria were observed between Melkassa and Alem Tena. In all the locations, farmers used intricate combinations of traits for selecting common bean varieties. However, pod load and filling, as well as yield, were cited as the most important traits in both gender groups and in all locations. In addition, marketability, seed size and seed colour were perceived to be important selection criteria for men in all locations. Marketability was less important for women farmers in Melkassa and Arsi Negele, whereas taste was ranked fourth. In Melkassa and Alem Tena, earliness and drought tolerance were cited as important traits for both gender groups. The women farmers at Melkassa ranked earliness as the third most important selection criteria, while in Alem Tena and Arsi Negele, these traits were not important in the selection of common bean varieties (Figure 4). Resistance to insects and diseases was an important criterion in Arsi Negele, but not in other locations. In general, women ranked the taste and cooking time as the top criteria for varietal choice, while men did not consider these traits to be important. On the other hand, pod clearance and plant stand were ranked by the men, but they were not perceived as important by the women in all the locations. Stem strength ranked differently across locations, while the suitability of straw was ranked only at Melkassa and Alem Tena.

\section{Farmers' variety selection}

The results of the participatory variety selection of 144 genotypes at three research stations revealed that there was considerable variation among entries, based on the farmers' selection criteria. The ten best genotypes from each station were selected by farmers. At Melkassa, the ten genotypes selected by both men and women farmers were dominated by the white small-seeded beans. Genotypes, such as Awash-1, Awash Melka, 211333 and RAZ-42, were the top selected genotypes, followed by small and medium-sized red beans SCR-15, 211323 and SCR-11 (Table 2). In addition, yellow and speckled bean types were also selected by both groups. Red-and white-seeded beans $(70 \%$ of selected genotypes) were most preferred by men, whereas yellow and white bean genotypes were selected by women. The commercial small white variety, Awash- 1, was the most preferred variety and KAT-B1 was the earliest genotype in the trial selected at Melkassa. In Alem Tena, the majority of the selected genotypes in both gender groups were small white beans, such as Awash-1, Awash Melka, 228812, 232196, 211347 and RAZ-40 and two yellow beans (NC-39, Wedo and Roba) (Table 2). The men selected six small white, two yellow (one small and one large), one speckled and one small red genotype. Genotypes selected by women farmers included six small white, four yellow (one small, one medium and two large) and one speckled coloured genotype. Awash1, Deme, Awash Melka and NC-39 were the top selected, and the most preferred genotype in Alem Tena. In Arsi Negele, on the other hand, all the selected genotypes were small red-seeded beans, with the exception of SCR-15 and NC-16, which are large-seeded (Table 2). The landrace 214663 was the most selected genotype by both gender groups, while 214663, 241734, NC-07 and SER-125 were the 
most preferred genotypes of men. Similarly, 230526, 214663, NC-12 and SER-125 were the four top-ranked genotypes by women farmers in Arsi Negele. In Arsi Negele, $40 \%$ of the selected genotypes were landraces and only one released variety (Nasir) was selected. The two best genotypes selected in Aris Negele (214663 and 241734) were landraces collected from the southern part of Ethiopia. However, the majority of the selected genotypes in Alem Tena and Melkassa were released varieties and breeding lines, respectively.

A comparison of farmers' selection with the field performances of the selected genotypes revealed that farmers in Arsi Negele gave more attention to yield and yield-related traits than biotic and abiotic stress factors. However, in Melkassa and Alem Tena, farmers leaned more towards insect resistance and drought tolerance traits in their varietal selection practice (Table 1). In general, genotypes that were selected by Arsi Negele farmers were late maturing, with long grain filling duration, but relatively high grain and biomass yielders. At the other two stations, farmers' selected white small-seeded genotypes which are highly resistant to bruchid (RAZ lines), based on their agronomic performance. Similarly, the line RAZ 42 was one of the varieties selected by the national bean research program and submitted to the national variety release committee for verification and release. In Melkassa and Aleme tena, farmers selected genotypes that showed a wider range of variation for all the traits, except for grain yield. However, the genotypes selected in Arsi Negele had the widest range of variation for grain yield (Table 3).

The grain yield and days to maturity of the farmers' selected genotypes at all locations are presented in Figures 5. A significant range of the variations for grain yield and maturity was recorded among the selected genotypes. Among the selected genotypes, KAT-B1 and Nasir were the earliest (76) and latest (106) genotypes to mature, respectively. In addition, the mean grain yield for selected genotypes ranged from $12.9 \mathrm{~g} /$ plants (KAT-B1) to 45.4 $\mathrm{g} /$ plants (Nasir).

\section{Post-harvest usage and problems}

Farmers at Melksassa and Alem Tena generally use beans as boiled grain (Nifro) and for stew (Wot). Red and yellow beans are primarily used for boiled grain and the white beans are used for stew. Yellow beans are considered very tasty and have a short cooking time. In Alem Tena, farmers also use speckled beans for consumption. In addition to boiled grains and stews, farmers in Arsi Negele use beans for making soup. In this region, yellow and speckled bean types are more preferred than the white ones. The red bean variety (Nasir) is the most marketable bean in the area. However, speckled and yellow beans have also recently been attracting the consumers' attention in the market.

Farmers in Melkassa and Alem Tena recognized bruchids as the most important storage pest, while farmers in Arsi Negele perceived bruchid as a less important problem. Farmers used several insect management practices, such as chemicals (Phostoxin and Malathion) and different cultural practices, such as mixing beans with ash and hot pepper powder to reduce the grain loss caused by the insect. Storing beans with ash and pepper powder hinders the mobility and oviposition of the insect. According to the farmers in Melkassa, Awash Melka had some tolerance, compared to other released varieties, but the variety has a low marketability in the area. Alem Tena farmers indicated that yellow beans are more susceptible to bruchid than red and white genotypes. Farmers at both locations sold their beans soon after harvest, to avoid losses due to bruchids.

\section{Discussion}

The common bean, because of its short life-cycle compared to cereal crops, is perceived by farmers as a food security crop. In the present study, male and female farmers were invited to assess and select the genotypes, based on their preferences in on-station trials at three sites. The farmers were well aware of the selection criteria and that the different areas had different selection criteria. The selection criteria were associated with the socio-cultural and agroecological conditions of the areas. McGuire (2007) also reported that, in a highly heterogeneous farming environment, the farmers' preference and varietal choice is a result of the interaction of the social, economic and environmental factors. Furthermore, in such diverse farming systems, farmers employ a wide range of criteria for selecting their preferred genotypes. In order to understand the farmers' preferences and to closely work with farmers, participatory studies are an essential component of a plant breeding program. Ceccarelli and Grando (2006) also reported that participatory research is important, in order to understand the traits or combinations of traits that are of interest to farmers.

The common bean genotypes selected by farmers varied amongst the three sites and the gender groups. The farmers' preference and selected genotypes at Melkassa and Alem Tena showed some level of similarity. This is mainly due to the fact that these two environments are both droughtprone areas. In these areas, farmers traditionally prefer small white-seeded bean varieties, such as Awash-1 and Awash Melka which were ranked as the best genotypes. These two varieties were released for the Central Rift Valley areas. Moreover, farmers in the Central Rift Valley produce white beans for export and Awash-1 is the dominant genotype for this purpose (Legesse et al., 2006; Assefa et al., 2004). In addition to the white beans, farmers at Melkassa and Alem Tena selected yellow, red and speckled beans of various seed sizes. The most recently-released yellow seeded variety, which was introduced from Kenya, gained popularity in the Melkassa area due to its extreme earliness. Arsi Negele, on the other hand, is situated in the midaltitude area of the southern Rift Valley region and has a relatively high rainfall climate. In this area, all the selected genotypes were small to medium-sized red cooking bean types. Farmers in this area produce the red beans for household consumption and the local markets (Ferris and Kaganzi, 2008; Asfaw et al., 2009, 2012; Rubyogo et al., 2010), although some red beans are informally exported to the regional markets of northern Kenya (Ferris and Kaganzi, 2008; Rubyogo et al., 2010). Similarly, Asfaw et al. (2012) also reported that the small red and black beans were the most preferred varieties in the southern part of Ethiopia, while the small white beans were rated poorly. Of the top ten red beans selected in Arsi Negele, the first three genotypes were landraces. Acc.no 214663 was the most preferred landrace, which was originally collected from the southern part of the country. At Arsi Negele, some farmers have started growing the speckled beans for both local consumption and the market. Although farmers have a strong preference towards red and white bean types in the 
Table 1. Rank of selection criteria used by men and women farmers at Melkassa, Alem Tena and Arsi Negele - $\quad$ indicates that the criteria are not ranked for that location or farmers group.

\begin{tabular}{|c|c|c|c|c|c|c|}
\hline \multirow[b]{3}{*}{ Selection criteria } & \multicolumn{6}{|c|}{ Rank } \\
\hline & \multicolumn{2}{|c|}{ Melkassa } & \multicolumn{2}{|c|}{ Alem Tena } & \multicolumn{2}{|c|}{ Arsi Negele } \\
\hline & Male & Women & Male & Women & Male & Women \\
\hline Pod load and filling & 1 & 1 & 1 & 1 & 1 & 1 \\
\hline Yield & 2 & 2 & 2 & 2 & 2 & 2 \\
\hline Drought tolerance & 7 & 6 & 7 & 9 & 10 & 10 \\
\hline Marketability & 3 & 9 & 3 & 3 & 5 & 7 \\
\hline Seed colour & 4 & 7 & 4 & 4 & 3 & 3 \\
\hline Seed size and shape & 5 & 8 & 5 & 5 & 4 & 5 \\
\hline Earliness & 6 & 3 & 6 & 6 & 8 & 8 \\
\hline Insect and disease resistance & 8 & - & 9 & - & 6 & 6 \\
\hline Taste & - & 4 & - & 7 & - & 4 \\
\hline Cooking time & - & 5 & - & 8 & - & - \\
\hline Plant stands & 13 & - & 13 & - & 9 & - \\
\hline Stem strength & 10 & 10 & 10 & 10 & 7 & 9 \\
\hline Pod clearance & 12 & - & 11 & - & 8 & - \\
\hline Suitability of straw & 9 & 11 & 8 & 11 & - & - \\
\hline
\end{tabular}

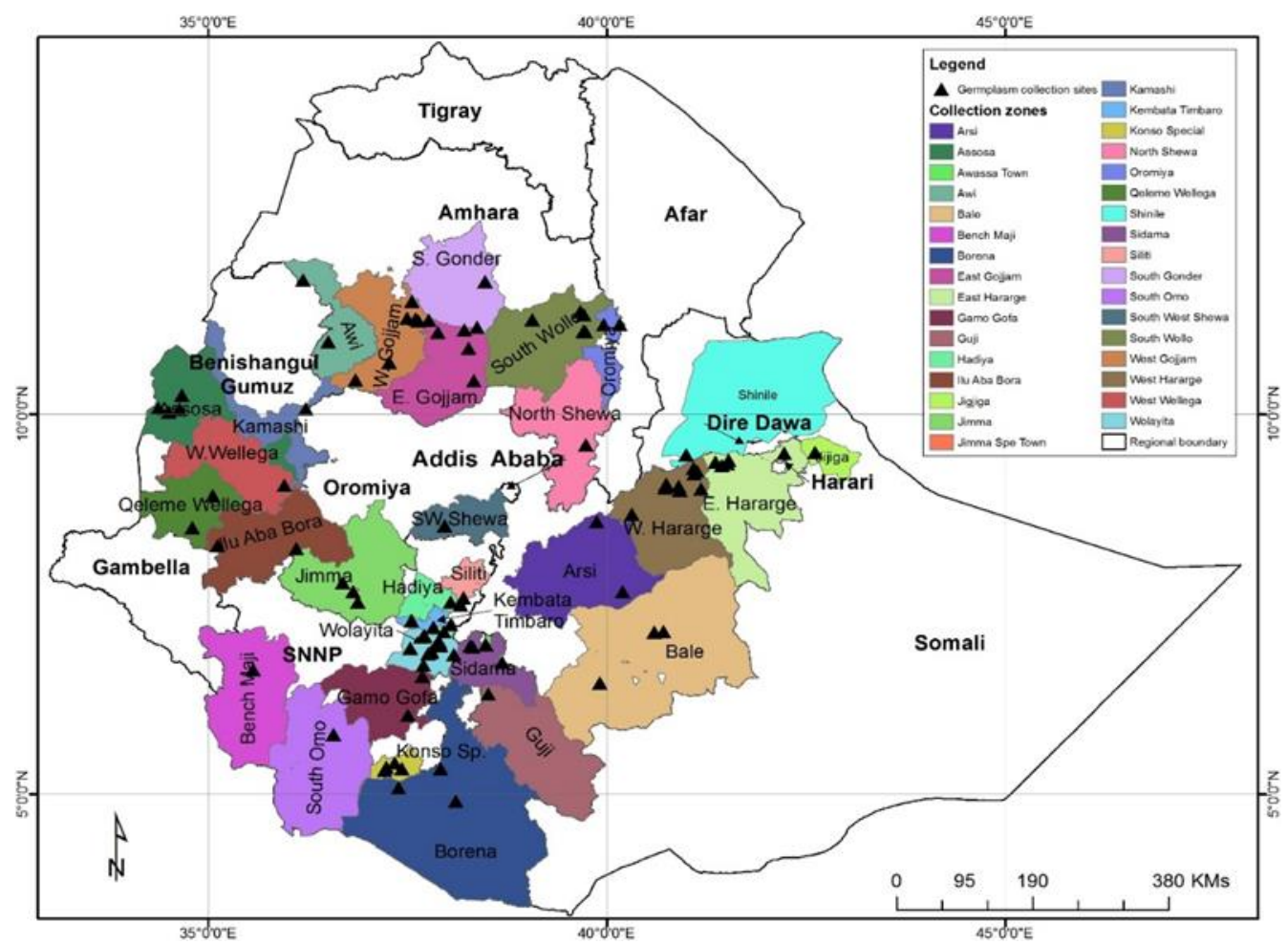

Fig 1. A map of Ethiopia showing the geographical positioning of the specific collection sites of 109 common bean landrace collections 
Table 2. Lists of 10 best selected genotypes by farmers at Melkassa, Alem Tena and Arsi Negele.

\begin{tabular}{|c|c|c|c|c|c|c|c|c|c|}
\hline \multirow[t]{2}{*}{ Rank } & \multicolumn{3}{|l|}{ Melkassa } & \multicolumn{3}{|l|}{ Alem Tena } & \multicolumn{3}{|c|}{ Arsi Negele } \\
\hline & Men & Women & All & Men & Women & All & Men & Women & All \\
\hline 1 & Awash-1 & NC-39 & Awash-1 & SCR-11 & Awash-1 & Awash-1 & 214663 & 230526 & 214663 \\
\hline 2 & Awash Melka & 230525 & NC-39 & NC-39 & Awash Melka & Deme & 241734 & 214663 & 241734 \\
\hline 3 & SCR-15 & Awash Melka & SCR-15 & Awash-1 & RAZ-42 & Awash Melka & NC-07 & NC-12 & NC-07 \\
\hline 4 & NC-39 & SCR-15 & Awash Melka & 211347 & Deme & NC-39 & 201066 & SER-125 & SER-125 \\
\hline 5 & 207934 & Deme & KAT-B1 & 228812 & Wedo & 228812 & SER-125 & SCR-15 & NC-12 \\
\hline 6 & KAT-B1 & KAT-B1 & 211333 & Deme & Roba & Wedo & 214665 & Nasir & SCR-15 \\
\hline 7 & 211333 & NC-15 & 211323 & 230661 & NC-39 & 232196 & NC-51 & 241734 & Nasir \\
\hline 8 & 211323 & NC-29 & Deme & Awash Melka & RAZ-40 & Roba & NC-12 & NC-07 & 201066 \\
\hline 9 & NC-30 & Awash-1 & SCR-11 & 232196 & NC-15 & 211347 & SCR-15 & NC-16 & 214665 \\
\hline 10 & SCR-11 & RAZ-42 & RAZ-42 & Roba & 228812 & RAZ-40 & Nasir & 201066 & NC-16 \\
\hline
\end{tabular}

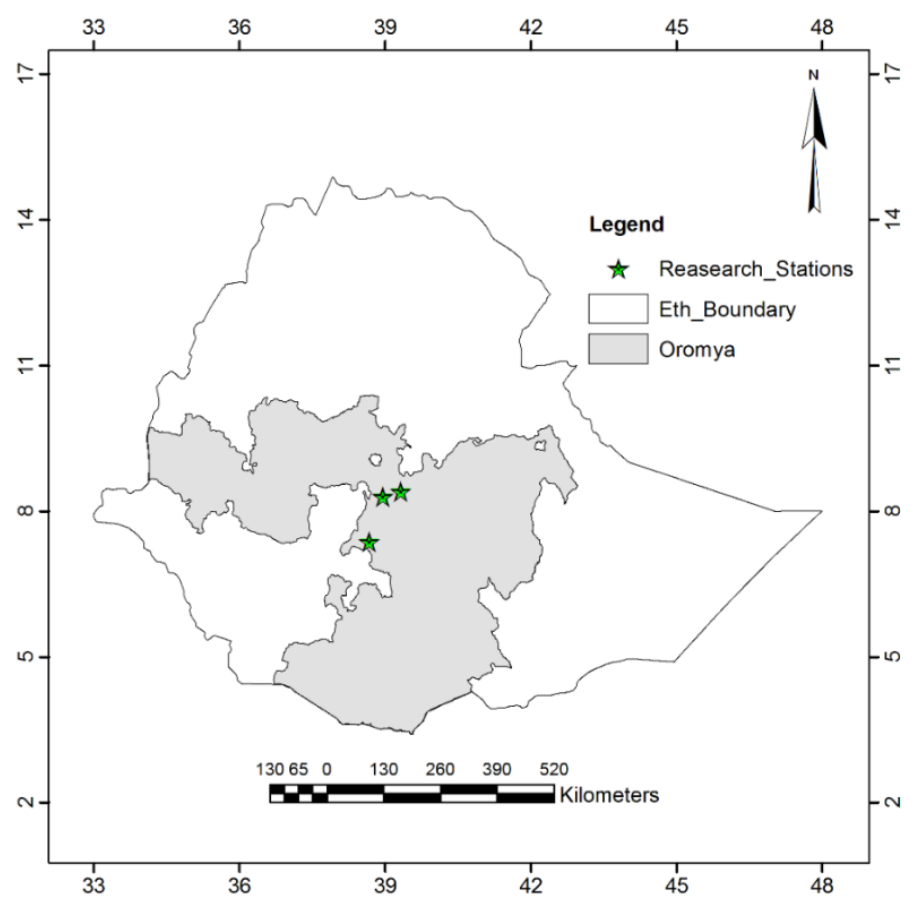

Fig 2. A map of Ethiopia showing the geographical positioning of the research stations used for participatory variety selection. 
Table 3. Seed colour, size, breeding status and means of farmers selected common bean genotypes for bruchid resistant and other agro-morphological traits measured under laboratory and field conditions.

\begin{tabular}{|c|c|c|c|c|c|c|c|c|c|c|c|c|}
\hline \multicolumn{13}{|c|}{ Melkassa } \\
\hline Genotype & SS & SC & Type & PAE & SWL & DTM & GFP & PPP & SPP & AGBM & GY & $\mathrm{HSW}$ \\
\hline Awash-1 & Small & White & VAR & 86.5 & 46.0 & 84.7 & 45.3 & 27.0 & 3.8 & 28.9 & 24.6 & 18.7 \\
\hline NC-39 & Medium & Yellow & LDR & 84.5 & 29.0 & 86.0 & 46.7 & 15.8 & 3.4 & 35.9 & 22.8 & 26.3 \\
\hline SCR-15 & Medium & Red & BRL & 82.5 & 16.5 & 85.0 & 45.0 & 27.7 & 3.7 & 32.2 & 30.3 & 38.4 \\
\hline Awash Melka & Small & White & VAR & 60.0 & 0.5 & 89.7 & 47.7 & 22.1 & 7.9 & 39.8 & 37.7 & 23.3 \\
\hline KAT-B1 & Large & Yellow & VAR & 80.0 & 26.0 & 76.0 & 40.3 & 11.9 & 3.4 & 23.6 & 12.9 & 42.6 \\
\hline 211333 & Small & White & LDR & 86.0 & 22.0 & 88.0 & 47.0 & 26.0 & 5.4 & 32.9 & 17.5 & 14.0 \\
\hline 211323 & Small & Red & LDR & 87.5 & 3.0 & 91.0 & 50.0 & 28.0 & 3.4 & 30.7 & 25.1 & 25.3 \\
\hline Deme & Large & Speckled & VAR & 66.5 & 28.0 & 87.3 & 44.0 & 17.1 & 3.8 & 43.0 & 39.3 & 59.9 \\
\hline SCR-11 & Medium & Red & BRL & 14.5 & 6.0 & 84.7 & 45.3 & 27.4 & 4.5 & 36.5 & 34.7 & 29.4 \\
\hline RAZ-42 & Small & White & RLN & 2.0 & 0.0 & 85.0 & 46.3 & 31.2 & 4.8 & 43.8 & 29.6 & 22.0 \\
\hline Mean & - & - & - & 65.0 & 18.0 & 85.7 & 45.8 & 23.4 & 4.4 & 34.7 & 27.5 & 30.0 \\
\hline SE & - & - & - & 1.9 & 0.9 & 4.1 & 2.5 & 6.4 & 1.4 & 6.4 & 8.6 & 13.5 \\
\hline CV\% & & & & 48.4 & 85.5 & 1.3 & 0.8 & 2.0 & 0.4 & 2.0 & 2.7 & 4.3 \\
\hline \multicolumn{13}{|c|}{ Aleme tena } \\
\hline Genotype & SS & $\mathrm{SC}$ & Type & PAE & SWL & DTM & GFP & PPP & SPP & AGBM & $\mathrm{GY}$ & $\mathrm{HSW}$ \\
\hline Awash-1 & Small & White & VAR & 86.5 & 46.0 & 86.7 & 43.7 & 28.2 & 4.1 & 29.7 & 25.0 & 18.0 \\
\hline Deme & Large & Speckled & VAR & 66.5 & 28.0 & 97.0 & 50.7 & 15.0 & 3.1 & 37.1 & 30.1 & 57.1 \\
\hline Awash Melka & Small & White & VAR & 60.0 & 0.5 & 90.0 & 44.3 & 39.3 & 5.4 & 50.2 & 37.0 & 21.5 \\
\hline NC-39 & Medium & Yellow & LDR & 84.5 & 29.0 & 86.7 & 44.0 & 20.2 & 6.1 & 37.8 & 25.7 & 25.4 \\
\hline 228812 & Small & White & LDR & 77.5 & 40.5 & 94.0 & 48.7 & 36.4 & 3.7 & 36.7 & 22.9 & 16.3 \\
\hline Wedo & Medium & Yellow & VAR & 89.5 & 18.0 & 81.0 & 40.3 & 17.0 & 4.1 & 34.1 & 24.3 & 32.8 \\
\hline 232196 & Small & White & LDR & 74.5 & 14.5 & 91.7 & 46.3 & 47.0 & 3.8 & 36.6 & 29.5 & 15.7 \\
\hline Roba & Small & Yellow & VAR & 49.5 & 22.0 & 93.7 & 46.7 & 35.8 & 5.0 & 44.1 & 29.9 & 18.1 \\
\hline 211347 & Small & White & LDR & 79.0 & 6.5 & 80.1 & 37.3 & 32.3 & 3.8 & 31.5 & 18.9 & 18.4 \\
\hline RAZ-40 & Medium & White & RLN & 0.0 & 0.0 & 88.7 & 47.0 & 22.1 & 3.4 & 39.2 & 33.6 & 37.8 \\
\hline Mean & - & - & - & 67.0 & 20.5 & 88.9 & 44.9 & 29.3 & 4.2 & 37.7 & 27.7 & 26.1 \\
\hline $\mathrm{SE}$ & - & - & - & 1.7 & 1.0 & 5.5 & 3.9 & 10.6 & 0.9 & 5.9 & 5.3 & 13.2 \\
\hline $\mathrm{CV} \%$ & - & - & - & 39.8 & 76.8 & 1.7 & 1.2 & 3.3 & 0.3 & 1.9 & 1.7 & 4.2 \\
\hline \multicolumn{13}{|c|}{ Aris Negele } \\
\hline Genotype & SS & SC & Type & PAE & SWL & DTM & GFP & PPP & SPP & AGBM & $\mathrm{GY}$ & $\mathrm{HSW}$ \\
\hline 214663 & Red & Small & LDR & 90.0 & 23.0 & 101.3 & 55.7 & 28.4 & 4.9 & 41.2 & 34.7 & 24.0 \\
\hline 241734 & Red & Small & LDR & 79.5 & 12.0 & 100.3 & 54.0 & 29.4 & 4.3 & 48.2 & 30.5 & 23.2 \\
\hline NC- 07 & Red & Small & LDR & 70.5 & 34.0 & 102.0 & 46.7 & 34.2 & 3.8 & 38.9 & 25.4 & 25.0 \\
\hline SER-125 & Red & Small & VAR & 91.0 & 26.5 & 102.0 & 55.7 & 23.2 & 5.3 & 36.4 & 33.4 & 24.5 \\
\hline NC-12 & Red & Small & LDR & 84.0 & 34.0 & 103.0 & 55.0 & 21.8 & 5.5 & 36.9 & 29.8 & 23.8 \\
\hline SCR-15 & Red & Medium & BRL & 82.5 & 16.5 & 103.0 & 56.3 & 28.7 & 3.6 & 46.3 & 32.6 & 38.5 \\
\hline Nasir & Red & Small & VAR & 83.5 & 43.0 & 105.7 & 60.7 & 42.6 & 3.8 & 50.0 & 45.4 & 23.5 \\
\hline 201066 & Red & Small & LDR & 84.5 & 9.5 & 99.0 & 53.3 & 21.9 & 6.3 & 44.6 & 30.9 & 23.5 \\
\hline 214665 & Red & Small & LDR & 93.5 & 29 & 102.7 & 57.0 & 31.5 & 5.0 & 39.2 & 30.7 & 21.6 \\
\hline NC-16 & Red & Small & LDR & 62.5 & 17.5 & 99.3 & 53.3 & 20.1 & 5.5 & 44.0 & 24.4 & 23.1 \\
\hline Mean & - & - & - & 82.0 & 24.5 & 101.8 & 54.8 & 28.2 & 4.8 & 42.6 & 31.8 & 25.1 \\
\hline SE & - & - & - & 0.6 & 0.7 & 2.0 & 3.6 & 6.9 & 0.9 & 4.8 & 5.8 & 4.8 \\
\hline CV\% & - & - & - & 11.6 & 43.8 & 0.6 & 1.1 & 2.2 & 0.3 & 1.5 & 1.8 & 1.5 \\
\hline
\end{tabular}

SS = seed size; $\mathrm{SC}=$ seed colour; PAE = percentage adult emergence; SWL = seed weight loss; DTM = days to maturity;

$\mathrm{GY}=$ grain yield 
A
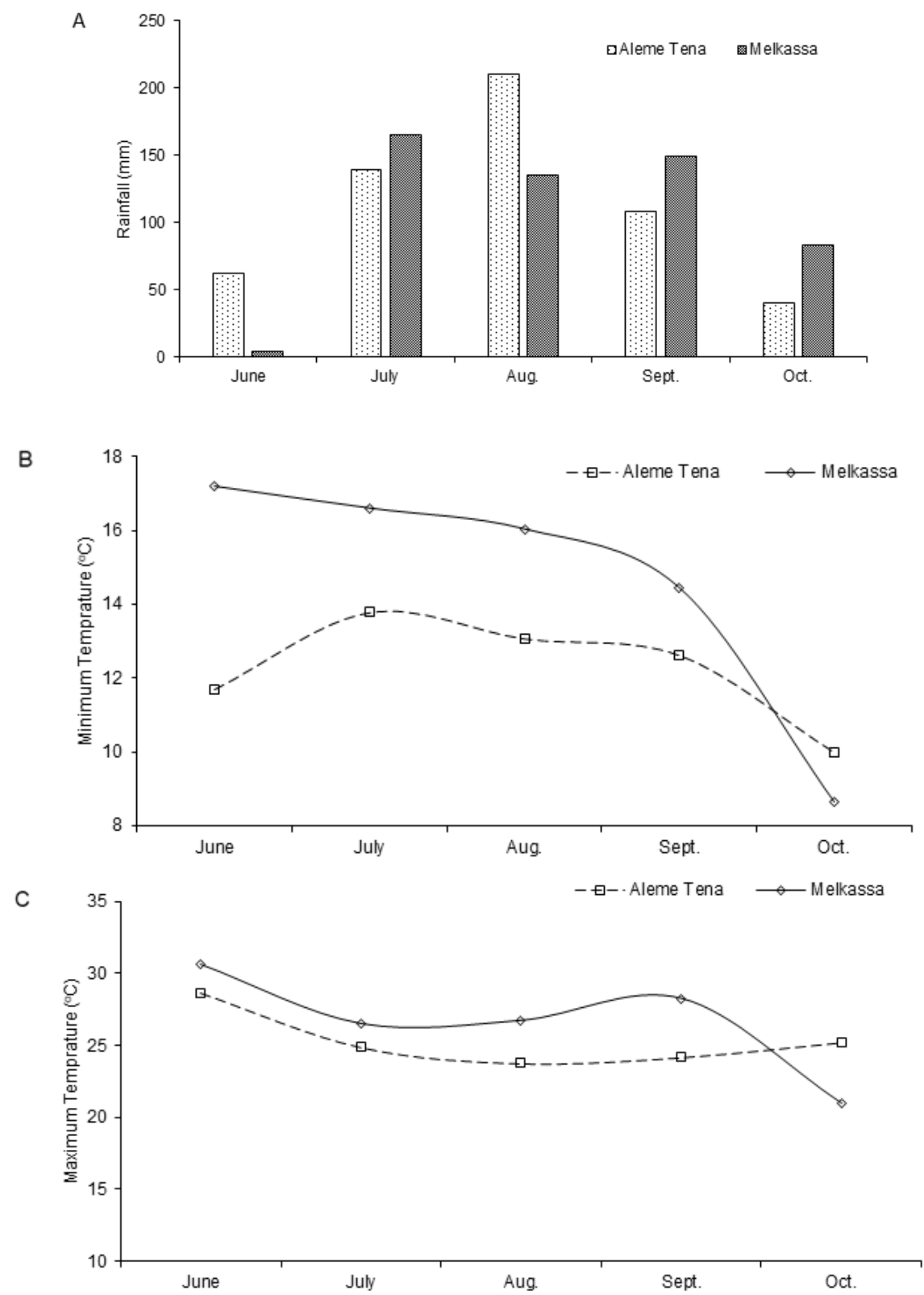

Fig 3. Climate data (A) rainfall (in $\mathrm{mm}$ ), (B) minimum and (C) maximum temperatures (in ${ }^{\circ} \mathrm{C}$ ) of Melkassa and Alem Tena sites during the growing.

study area, farmers grow several bean genotypes for multiple household uses. This suggests that farmers are flexible and willing to produce a range of common bean types, in addition to the well-acquainted white and red small- seeded bean varieties.

Varietal choice and related selection criteria may vary for different groups of farmers (de Boef and Thijssen, 2006). Gender is one major social category in which variation can be expected, in this regard. Women and men have different gender roles and responsibilities in their society, on the farm and in the household. Consequently, these roles affect their decision to choose a variety or sets of varieties. Both gender groups had a similar preference for yield and yield- related traits and a variety with high-grain yield is obviously preferred by both men and women. In the present study, all farmers who participated in the PVS ranked yield and pod load and filling as their top selection criteria in all the locations. A similar result from a PVS was reported for bean genotypes by other researchers in the Central Rift Valley and eastern parts of Ethiopia (Mekbib, 1997; Assefa et al., 2005, 2014).

[However, differences were observed between men and women for other selection criteria. Men tended to focus more on seed size, seed colour and market-related traits. The tendency towards seed colour and size is highly influenced by market preferences in the different locations. However, women are generally more interested in postharvest processing and food preparation aspects. Women have an important role in the assessment of postharvest qualities, such as taste, cooking quality and time. On the other hand, men totally ignored the culinary quality of beans. Assefa et al. (2014) also reported on the variation between the gender groups with regard to market and userelated traits. This signifies the importance of involving participants from different farming systems and farmers' groups in the participatory studies. In Arsi Negele, diseases 
and insects are the most prevalent production constraints. The pressure of disease is generally high, compared to the other locations, due to the high rainfall in the area. Arsi Negele farmers totally ignored the cooking time and the suitability of straw, as selection criteria because fuel wood and other forage crops are available in abundance.

The choice of selection criteria was significantly associated with the prevalent environmental conditions. In areas where drought and disease problems are prevalent, men and women farmers tend to have similar preferences. In Melkassa and Alem Tena, drought-related traits, such as earliness and drought tolerance were among the top listed traits, whereas in Arsi Negele, disease and insect resistance was more vital. Melkassa and Alem Tena are characterized by low rainfall of a short duration. In these areas, farmers consider earliness and drought tolerance as important traits for selecting bean varieties (Asfaw et al., 2012). Although the yield potential of KAT-B1 is low, farmers selected it because of its earliness, as the common bean is the first food available for the household in drought-prone areas in central Rift Valley. Women farmers prefer early genotypes to the high yielding late maturing genotypes, in order to fulfill the food needs of the household (Assefa et al., 2014).

Although the storage insect pests were found to be the major problem in all locations, the problem is more severe in Melkassa and Alem Tena than in Arsi Negele. This may be related to the favorable environmental conditions for the growth and the development of the insect at the two sites, compared to the cool and humid Arsi Negele. Farmers were able to recognize the good tolerance level of the released variety Awash Melka, to the insect. This fact was also confirmed in our laboratory analysis.

\section{Materials and methods}

\section{Plant materials}

On the basis of their level of resistance, population structure and genetic distances, a total of 144 genotypes were selected. The selected common bean genotypes comprised of 109 landraces, 16 released varieties and 19 pre-release breeding lines. The 109 common bean landraces were collected from different regions of Ethiopia (Fig 1). Of the 18 pre-released genotypes, 15 genotypes were resistant to the Mexican bean weevil. The inclusion of landraces and prereleased varieties allows farmers to have more options and it allows them, to compare these genotypes with the released commercial varieties. This avoids the risk of the failure of adoption and allows the breeder to include the farmers' preferred traits in their breeding program. A list of the tested genotypes is given in supplementary table 1 . The 144 genotypes were planted in a $12 \times 12$ row-column design, with three replications. The common bean genotypes were planted in one row of $3 \mathrm{~m}$ long, with an inter-row spacing of $60 \mathrm{~cm}$ and an intra-row spacing of $40 \mathrm{~cm}$. Weeds were controlled with frequent hand- weeding throughout the experiment. Di-ammonium phosphate (DAP) fertilizer was applied during planting, at a rate of $100 \mathrm{~kg} / \mathrm{ha}$ (Assefa et al., 2014) and other agronomic practices were done as per recommendation for each site.

\section{Description of the study site}

The study was conducted at three on-station trial sites in the Oromiya region of central Ethiopia (Fig 2). The three sites were Melkassa $\left(8^{0} 24^{\prime} 52.04 " \mathrm{~N}, 39^{0} 19^{\prime} 41.22 " \mathrm{E}, 1550\right.$ m.a.s.I.), Alem Tena ( $8^{\circ} 17^{\prime} 32.29^{\prime \prime} \mathrm{N}, 38^{0} 56^{\prime} 48.77^{\prime \prime E}, 1611$ m.a.s.l,) and Arsi Negele $\left(7^{0} 22^{\prime} 30.29^{\prime \prime} \mathrm{N}, 38^{\circ} 40^{\prime} 17.78^{\prime E} \mathrm{E}, 1960\right.$ m.a.s.I). This study was carried out in the main cropping season of 2014. The climatic data of Melkassa and Alem Tena were collected from the Melkassa and Debrie Zeit Agricultural research centers, respectively. The climatic data on rainfall and temperature for only the two sites is presented in Fig 3 . The soil types of Melkassa and Alem Tena are sandy and loamy, while the soil in Arsi Negele is clay.

\section{Data collection and analysis}

The participating farmers were selected, based on their indigenous knowledge, of bean production and their willingness to participate in the variety evaluation. The selection of participants was made with the help of development extension agents and technical assistants from each station. From each site, 20 (10 male and 10 female) common bean-producing farmers were selected. A visual evaluation of the genotypes was made when the crop was at the late pod filling and maturity stage. Focus group discussions were conducted to identify the common bean production constraints at each location. Local languages were used, to enable farmers to express their ideas easily during the discussion time.

The participating farmers were divided into male and female groups to explore the differences in the selection criteria between the two groups. PVS was applied to select common bean genotypes that possess the farmers' preferred traits and to facilitate the selection of parental genotypes for breeding for bruchid resistance (Zabrotes subfasciatus). Initially, farmers were allowed to discuss and agree on criteria that they thought were important for selecting a given variety for the different groups. Subsequently, the evaluation procedure was explained to the participating farmers and scoring was done individually. Four plastic tags, each with a different colour were given to the farmers, to facilitate the selection process. Plastic bags were put in each line in the field and farmers put the different coloured tags inside the plastic bags, based on their preferences. Seeds of each genotype were also displayed to the farmers, in order for the participants to observe the seed colours and sizes. The number of tags from each plastic bag from each genotype were counted. Immediately after the field evaluation, the best and the worst selected genotypes were identified and group discussions were held in the field, to rank the selection criteria of each group.

\section{Conclusion}

In the present study, the farmers' most preferred traits and genotypes were identified. The selection criteria included yield, pod load and filling, drought tolerant, seed colour, size and shape, earliness, drought tolerance, insect and disease resistance, taste and cooking time. The relative importance of the selection criteria varies from location to location, and among farmer groups. The variation in the selection criteria is highly influenced by socio-cultural, economic and agroecological factors. The study confirmed that both men and women need to be involved in identifying farmers' preferences, setting priorities and re-orienting research directions. Understanding of varietal trait preferences across farming systems and farmer groups, will provide new insights for breeders to anticipate which traits and trait 
combinations can benefit the target farming system or farmer group. This can be done through a participatory variety selection that enhances the development of demand-driven, client- oriented crop technologies, dissemination and adoption. The information generated from this study can be utilized by plant breeders for the incorporation of farmers preferred traits into the beans breeding program.

\section{Acknowledgments}

The authors wish to acknowledge the Alliance for Green Revolution in Africa (AGRA) for financial support and Melkassa Agricultural Research Centre (MARC) for providing the necessary material and technical support during the study period.

\section{Reference}

Asfaw A, Almekinders CJM, Blair MW, Struik PC (2012) Participatory approach in common bean (Phaseolus vulgaris L.) breeding for drought tolerance for southern Ethiopia. Plant Breed. 131: 125-134.

Asfaw A, Blair MW, Almekinders C (2009) Genetic diversity and population structure of common bean (Phaseolus vulgaris L.) landraces from the East African highlands. Theor Appl Genet. 120: 1-12.

Assefa T, Abebe G, Fininsa C, Tesso B, Al-Tawaha ARM (2005) Participatory bean breeding with women and small holder farmers in Eastern Ethiopia. World J Agric Sci. 1: 28-35.

Assefa T, Reda F, Amsalu B, Abate T (2006) Integrated approach for the promotion of common beans for export. In proceedings of first international conferences for scaling up/out of technologies, 10-15. Addis Ababa: Ethiopian Institute of Agricultural Research (EIAR).

Assefa T, Sperling L, Dagne B, Argaw W, Tessema D, Beebe S, (2014) Participatory plant breeding with traders and farmers for white pea bean in Ethiopia. J Agric Educ Exten. 20: 497-512.

De Boef WS, Thijssen MH (2006) Participatory tools working with crops, varieties and seeds. A guide for professionals applying participatory approaches in agro biodiversity management, plant breeding and seed sector development. Wageningen, Wageningen International.

Buruchara R, Chirwa R, Sperling L, Mukankusi C, Rubyogo JC, Muthoni R, Abang MM (2011) Development and delivery of bean varieties in Africa: the pan- Africa bean research alliance (PABRA) model. Afr Crop Sci J. 19: 227-245.

Ceccarelli S, Grando S (2006) Decentralized-participatory plant breeding. p. 145-156. In Tuberosa R, Phillips R, Gale $M$. (eds.) The wake of the double helix: from the green revolution to the gene revolution. Avenue media, Bologna, Italy.
Ceccarelli S, Grando S (2007) Decentralized-participatory plant breeding: an example of demand driven research. Euphytica. 155: 349-360.

Ceccarelli S, Grando S, Tutwiler R, Baha J, Martin AM, Salahieh H, Goodchild A, Michael MJ (2000) A methodological study on participatory barley breeding: I. Selection phase. Euphytica. 111: 81-104.

CIAT (2013) Delivering on the promise of tropical agriculture. Cali, Colombia.

CSA (2015) Report on area and production of crops: Agricultural sample survey on private peasant holdings of 2014/2015 Meher season. Central Statistic Authority, Addis Ababa, Ethiopia.

Dawit A, Spielman DJ (2010) The Ethiopian seed system: regulations, institutions and stakeholders. In paper submitted for ESSP Policy Conference 2006 "Bridging, balancing, and scaling up: Advancing the rural growth agenda in Ethiopia." Addis Ababa, Ethiopia.

Ferris S, Kaganzi E (2008) Evaluating marketing opportunities for haricot beans in Ethiopia. Improving productivity and market success (IPMS) of Ethiopian farmer's project working paper 7. International Livestock Research Institute (ILRI), Nairobi, Kenya.

Katungi E, Farrow A, J Chianu, Sperling L, Beebe S (2009) Common bean in Eastern and Southern Africa: a situation and outlook analysis. International Center for Tropical Agriculture (CIAT), Kampala, Uganda.

Legesse D, Kumssa G, Assefa T, Taha M, Gobena J, Alema T, Abebe A, Mohhamed Y, Terefe $H$ (2006) Production and marketing of white pea beans in the Rift Valley, Ethiopia. National bean research program of the Ethiopian Institute of Agricultural Research.

McGuire SJ (2007) Vulnerability in farmer seed systems: Farmer practices for coping with seed insecurity for sorghum in Eastern Ethiopia. Econ Bot. 61: 211-222.

Mekbib $F$ (1997) Farmer participation in common bean genotype evaluation: The case of Eastern Ethiopia. Exper Agric. 33: 399-408.

Pan Africa Bean Research Alliance (2005) Annual Report 2005. Kampala, Uganda.

Rubyogo JC, Gebeyehu S, Tumsa K, NegasK, Habte E, Katungi E, Sperling L, Wozemba D (2011) Increased bean productivity through increased access to improved seeds and use of improved bean management techniques in Ethiopia.

Rubyogo JC, Sperling L, Muthoni R, Buruchara R (2010) Bean seed delivery for small farmers in sub-Saharan Africa: The power of partnerships. Soc Nat Res. 23: 1-18.

Sperling L, Ashby JA, Smith ME, Weltzien E, McGuire S (2001) Participatory plant breeding: A Framework for analyzing diverse approaches. Euphytica. 122: 439-450.

Sperling L, Loevinshohn M (1996) Using diversity: Enhancing and maintaining genetic resources on farm. Proceding workshop held on 19-21 June 1995, New Delhi. India. IDRC: New Delhi. 